Rapid Reviews COVID-19•

\title{
Review 2: "Small-molecule ligands can inhibit -1 programmed ribosomal frameshifting in a broad spectrum of coronaviruses"
}

Yitzhak Pilpel ${ }^{1}$

${ }^{1}$ Weizmann

Published on: Dec 05, 2021

DOI: $10.1162 / 2 \mathrm{e} 3983 f 5.90 c 7 e 7 d e$

License: Creative Commons Attribution 4.0 International License (CC-BY 4.0). 


\section{RR:C19 Evidence Scale rating by reviewer:}

- Strong. The main study claims are very well-justified by the data and analytic methods used. There is little room for doubt that the study produced has very similar results and conclusions as compared with the hypothetical ideal study. The study's main claims should be considered conclusive and actionable without reservation.

$* * * * * * * * * * * * * * * * * * * * * * * * * * * * * * * * * * * * * * * *$

\section{Review:}

Munshi et al. present a drug screen for SARS-CoV-2 and other coronaviruses. Their strategy is based on a conserved -1 Programmed Ribosomal Frameshift (PRF) common to many coronaviruses. They used a collection of approved drugs in a cell-free assay and then in human cell lines to look for drugs that inhibit PRF. They used the wellestablished dual reporter assay for quantifying -1 PRF. The authors used other viruses, like HIV, as a negative controls or specificity checks to look for drugs that specifically inhibit -1 PRF in coronaviruses. They did a thorough bioinformatics analysis to select representatives of the virus from several evolutionary clades and tested some from each. Curiously, different coronaviruses have different RNA secondary structures, hence the mechanism that governs $-1 \mathrm{PRF}$.

The authors found several drugs with potent and specific effects. These inhibitrather specifically--1 PRF in several of the coronaviruses but not in the negative controls. Interestingly, different drugs showed specificity to different -1 PRF mechanisms. Results from cell-free assays were followed up on in cell line assays using human cells. The work was done with rigor, thoroughness, and with up-to-date methods and technologies from a diverse set of fields. The results are promising; these could develop into much-needed drug candidates.

The only suggestion I have is that the connection between viral clade to the secondary structure at the -1 PRF sites and the drug be made clearer. Fig 1 does show the first two, but it is hard to reveal which secondary structure and -1 PRF mechanism appear in which viral clade (i.e. are there clades with more than one mechanism, or are there mechanisms that appear in more than one clade?). Do different drugs have specificity that depends on a mechanism and on RNA secondary structure (or perhaps on clade affiliation?). 